\title{
The Galactic Center Region Imaged by VERITAS from 2010-2012
}

\author{
Matthias Beilicke $^{1}$ for the VERITAS Collaboration ${ }^{2}$ \\ ${ }^{1}$ Department of Physics and McDonnell Center for the Space Sciences, Washington University, St. Louis, MO, USA \\ ${ }^{2}$ http://veritas.sao.arizona.edu/ \\ Corresponding author: beilicke@physics.wustl.edu
}

\begin{abstract}
The galactic center has long been a region of interest for high-energy and very-high-energy observations. Many potential sources of $\mathrm{GeV} / \mathrm{TeV} \gamma$-ray emission are located in this region, e.g. the accretion of matter onto the central black hole, cosmic rays from a nearby shell-type supernova remnant, or the annihilation of dark matter. The galactic center has been detected at $\mathrm{MeV} / \mathrm{GeV}$ energies by EGRET and recently by Fermi/LAT. At TeV energies, the galactic center was detected at the level of 4 standard deviations with the Whipple $10 \mathrm{~m}$ telescope and with one order of magnitude better sensitivity by H.E.S.S. and MAGIC. We present the results from 3 years of VERITAS galactic center observations conducted at large zenith angles. The results are compared to astrophysical models.
\end{abstract}

Keywords: gamma-rays - galactic center - black hole - non-thermal - VERITAS.

\section{Introduction}

The center of our galaxy harbors a $4 \times 10^{6} M_{\odot}$ black hole $(\mathrm{BH})$ coinciding with the strong radio source Sgr A*. $\mathrm{X}$-ray $/ \mathrm{MeV} / \mathrm{GeV}$ transients in this region are observed on a regular basis. Various astrophysical sources located close to the galactic center (GC) may potentially be capable of accelerating particles to multi-TeV energies, such as the supernova remnant Sgr A East or a pulsar wind nebula [1]. Furthermore, super-symmetric neutralinos $\chi$ are discussed as potential candidates for dark matter accumulating in the GC region and annihilating into $\gamma$-rays [2]. The resulting spectrum would have a cut-off near the neutralino mass $m_{\chi}$. Assuming a certain dark matter density profile the expected $\gamma$-ray flux along the line-of-sight integral can be calculated as a function of $m_{\chi}$ and the annihilation cross section [3] and can in turn be compared to measured upper limits.

EGRET detected a $\mathrm{MeV} / \mathrm{GeV}$ source 3EG J17462851 coincident with the GC position [4] and recently Fermi/LAT resolved several sources in the GC region [5], see Fig. 3. However, uncertainties in the diffuse galactic background models and limited angular resolution at $\mathrm{MeV} / \mathrm{GeV}$ energies make it difficult to study the morphologies of these sources. At $\mathrm{GeV} / \mathrm{TeV}$ energies a detection from the direction of the GC was first reported in 2001/02 by the CANGAROO II collaboration with a steep energy spectrum $\mathrm{dN} / \mathrm{dE} \propto \mathrm{E}^{-4.6}$ at the level of $10 \%$ of the Crab Nebula flux [6]. Shortly after, evidence at the level of 3.7 standard deviations (s.d.) was reported from the Whipple $10 \mathrm{~m}$ collabora- tion [7]. The $\mathrm{GC}$ was finally confirmed as a $\mathrm{GeV} / \mathrm{TeV}$ $\gamma$-ray source by the H.E.S.S. collaboration [8] (the position of the supernova remnant Sgr A East could be excluded as the source of the $\gamma$-ray emission). The energy spectrum measured by H.E.S.S. is well described by a power-law $\mathrm{dN} / \mathrm{dE} \propto \mathrm{E}^{-2.1}$ with a cut-off at $\sim 15 \mathrm{TeV}$. The H.E.S.S. observations revealed a diffuse $\mathrm{GeV} / \mathrm{TeV}$ $\gamma$-ray component (dashed contour lines in Fig. 3) which is aligned along the galactic plane and follows the structure of molecular clouds [9]; the emission is explained by an interaction of local cosmic rays (CRs) with matter of the molecular clouds. The MAGIC collaboration detected the GC (7 s. d.) in 2004/05 observations performed at large zenith angles (LZA) [10], followed by a strong (> 10 standard deviations) VERITAS LZA detection in 2010 [11].

\section{VERITAS Observations of the Galactic Center}

GC observations Due to its declination the GC can only be observed by VERITAS at LZA (zenith angles $60-66 \mathrm{deg}$ ) - strongly decreasing the angular resolution and sensitivity. The use of the displacement parameter [12], between the center of gravity of the image and the shower position, has been used in the VERITAS event reconstruction which strongly improved the sensitivity for LZA observations [11]. The performance and energy reconstruction have been confirmed on LZA Crab Nebula data. The column density of the atmosphere changes with $1 / \cos (z)$. In a conservative es- 
timate, the systematic error in the energy/flux reconstruction can be expected to scale accordingly. More detailed studies are needed for an accurate estimate; for the GC observations we currently give a conservative value of a systematic error on the LZA flux normalization of $\Delta \Phi / \Phi \simeq 0.4$. The GC was observed by VERITAS in 2010-2012 for $46 \mathrm{hrs}$ (good quality data, dead-time corrected) with an average energy threshold of $E_{\mathrm{thr}} \simeq 2.5 \mathrm{TeV}$.

GC results The VERITAS sky map of the GC region is shown in Fig. 3. An 18 s.d. excess is detected. No evidence for variability was found in the 3-year data. The energy spectrum is shown in Fig. 1 and is found to be compatible with the spectra measured by Whipple, H.E.S.S., and MAGIC. Since the large LZA effective areas of the VERITAS observations compensate a shorter exposure of low-zenith observations, the statistical errors of the $E>2.5 \mathrm{TeV}$ data points are comparable or even smaller than those of the H.E.S.S. measurements.

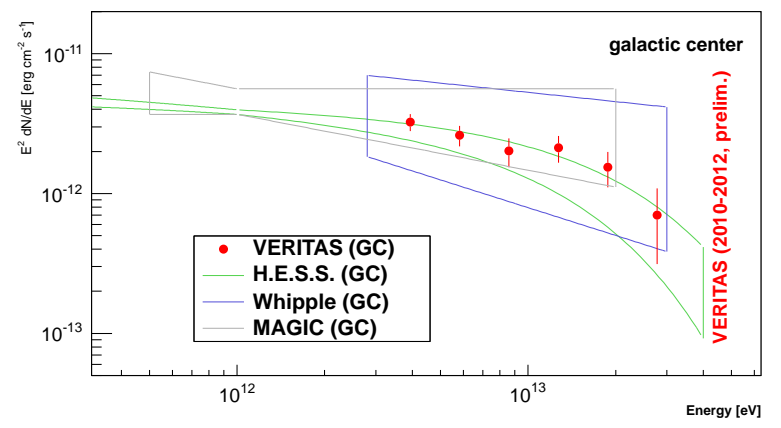

Figure 1: VERITAS energy spectrum measured from the direction of the GC (statistical errors only). Also shown are bow ties representing the spectra measured by Whipple [7], H.E.S.S. [8], and MAGIC [10].

Diffuse flux limit and dark-matter annihilation OFF-source observations were performed in a field located in the vicinity of the GC region (similar zenith angles and sky brightness) without a known $\mathrm{TeV} \gamma$-ray source. These observations are used to study the background acceptance throughout the field of view and allow the estimate of a diffuse $\gamma$-ray component surrounding the position of the GC. An upper limit of the diffuse $\gamma$-ray flux can in turn be compared with line-of-sight integrals along the density profile $\int \rho^{2} \mathrm{dl}$, in order to constrain the annihilation cross section for a particular dark matter model, dark matter particle mass and density profile $\rho(r)$. Due to its likely astrophysical origin the excess at the GC itself, as well as a region along the galactic plane, will be excluded from this analysis (work in progress).
Hadronic models Hadronic acceleration models [13, 14] involve: (i) hadrons being accelerated in the $\mathrm{BH}$ vicinity (few tens of Schwarzschild radii). (ii) The accelerated protons diffuse out into the interstellar medium where they (iii) produce neutral pions which decay into $\mathrm{GeV} / \mathrm{TeV} \gamma$-rays. Linden et al. (2012) discuss the surrounding gas as proton target defining the morphology of the TeV $\gamma$-ray emission [15]. Changes in $\gamma$-ray flux in those models can be caused by changing conditions in the $\mathrm{BH}$ vicinity (e.g. accretion). The time scales of flux variations are $\sim 10^{4}$ yr at $\mathrm{MeV} / \mathrm{GeV}$ energies (old flares) and $\sim 10$ yr at $E>10 \mathrm{TeV}$ ('new' flares caused by recently injected high-energy particles) [13]. Constraining the $E>10 \mathrm{TeV}$ spectral variability would serve as an important test for this class of models.

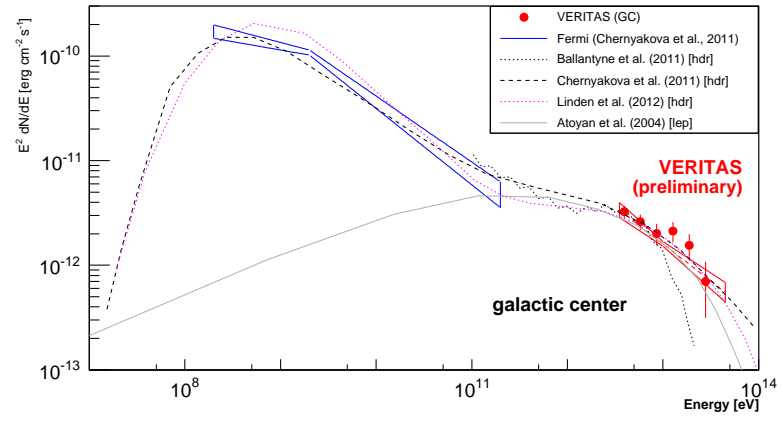

Figure 2: VERITAS energy spectrum compared to hadronic [13, 14, 15] and leptonic [16] emission models discussed for the GC source. The Fermi/LAT bow tie is taken from [13].

Leptonic models Atoyan et al. (2004) [16] discuss a $\mathrm{BH}$ plerion model in which a termination shock of a leptonic wind accelerates leptons to relativistic energies which in turn produce $\mathrm{TeV} \gamma$-rays via inverse Compton scattering. The flux variability time scale in this model is on the order of $T_{\text {var }} \sim 100 \mathrm{yr}$. The hadronic and the leptonic models are shown together with the VERITAS/Fermi data in Fig. 2. The leptonic model clearly fails in explaining the flux in the $\mathrm{MeV} / \mathrm{GeV}$ regime. However, this emission may well originate from a spatially different region or mechanism other than the $\mathrm{TeV}$ $\gamma$-ray emission. The hadronic models can explain the SED by the superposition of different flare stages. Future Fermi/VERITAS flux correlation studies, as well as the measurement of the TeV energy cut-off and limits on the $E>10 \mathrm{TeV}$ variability will serve as crucial inputs for the modeling.

\section{Summary and Conclusion}

VERITAS is capable of detecting the GC within 3 hrs in observations conducted at zenith angles greater than 


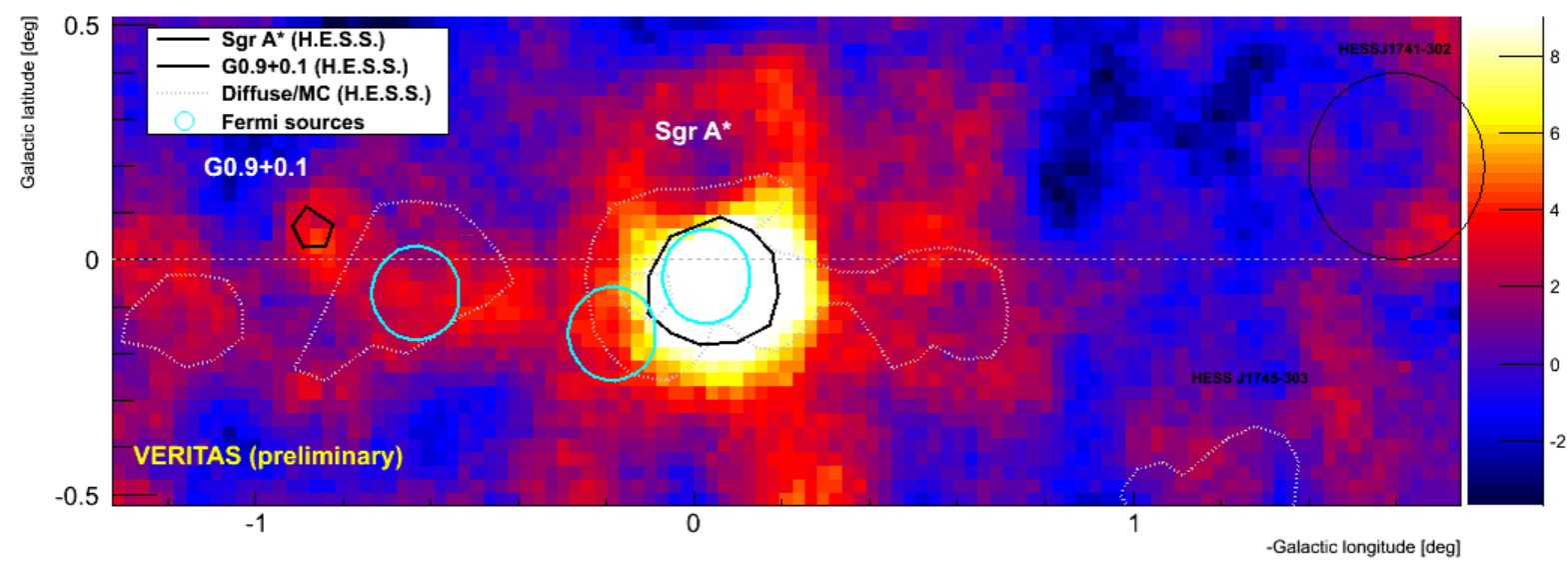

Figure 3: VERITAS sky map of the GC region (smoothed excess significances, ring background, scale truncated). The black contour lines indicate the GC and the supernova remnant G 0.9+0.1 as seen by H.E.S.S. [8]. The gray dashed lines indicate the H.E.S.S. diffuse emission along the galactic plane and from HESS J1745-303 [9]. The position of HESS J1741-302 is indicated, as well (circle); the flux/spectrum of this source make it very unlikely to be detected in VERITAS LZA observations. The solid circles (cyan color) indicate the positions of the $\mathrm{MeV} / \mathrm{GeV}$ sources taken from the second Fermi/LAT catalog [5].

$60 \mathrm{deg}$. The measured energy spectrum is found to be in agreement with earlier measurements by H.E.S.S., MAGIC, and Whipple. Future observations to measure the cut-off energy in the spectrum and to determine limits on the flux variability at the highest energies will place constraints on emission models. The recently discovered giant molecular cloud heading towards the immediate vicinity of the GC BH [17] represents further motivation for future $\mathrm{TeV} \gamma$-ray monitoring of this region. An upper limit on diffuse $\gamma$-ray emission and, in consequence, a limit on the photon flux initiated by the annihilation of dark matter particles is work in progress.

\section{References}

[1] Q.D. Wang, F.J. Lu, E.V. Gotthelf, et al., MNRAS 367, 937 (2006). doi:10.1111/j.1365-2966.2006.09998.x

[2] G. Jungman, M. Kamionkowski, \& K. Griest, PhR 267, 195 (1996).

[3] L. Bergström, P. Ullio, \& J. Buckley, APh 9, 137 (1998).

[4] R.C. Hartman, D.L. Bertsch, S.D. Bloom, et al., ApJS 123, 79 (1999).

[5] A.A. Abdo, et al., ApJS 188, 405 (2010). doi:10.1088/0067-0049/188/2/405

[6] K. Tsuchiya, R. Enomoto, L.T. Ksenofontov, et al., ApJ 606, L115 (2004). doi:10.1086/421292

\section{Acknowledgement}

This research is supported by grants from the U.S. Department of Energy Office of Science, the U.S. National Science Foundation and the Smithsonian Institution, by NSERC in Canada, by Science Foundation Ireland (SFI 10/RFP/AST2748) and by STFC in the U.K. We acknowledge the excellent work of the technical support staff at the Fred Lawrence Whipple Observatory and at the collaborating institutions in the construction and operation of the instrument.

[7] K. Kosack, H.M. Badran, I.H. Bond, et al., ApJ 608, 97 (2004). doi:10.1086/422469

[8] F.A. Aharonian, et al., A\&A 425, L13 (2004).

[9] F.A. Aharonian, et al., Nature 439, 695 (2006). doi:10.1038/nature04467

[10] J. Albert, et al., ApJ 638, L101 (2006). doi:10.1086/501164

[11] M. Beilicke, et al. (VERITAS collaboration), Proc. 2011 Fermi Symp., arXiv 1109.6836 (2011).

[12] J.H. Buckley, C.W. Akerlof, D.A. Carter-Lewis, et al., A\&A 329, 639 (1998).

[13] M. Chernyakova, D. Malyshev, F.A. Aharonian, et al., ApJ 726, 60 (2011). doi:10.1088/0004-637X/726/2/60 
[14] D.R. Ballantyne, M. Schumann, \& B. Ford, MN- [16] A. Atoyan, \& C.D. Dermer, ApJ 617, L123 (2004). RAS 410, 1521 (2011). doi:10.1086/427390

[15] T. Linden, E. Lovegrove, \& S. Profumo, arXiV 1203.3539 (2012).

[17] S. Gillessen, R. Genzel, T. Fritz, et al., Nature 481, 51 (2012). doi:10.1038/nature10652 